\title{
Refined forms of Oppenheim and Cusa-Huygens type inequalities
}

\author{
Yogesh J. Bagul and Christophe Chesneau
}

\begin{abstract}
We refine Oppenheim's inequality as well as generalized Cusa-Huygens type inequalities established recently by some researchers. One of the results where the bounds of $\sin x / x$ are tractable will be used to obtain a sharp version of Yang's inequality.
\end{abstract}

\section{Introduction}

The famous Cusa-Huygens inequality $[7,12,16-18]$ is known as

$$
\frac{\sin x}{x}<\frac{2+\cos x}{3}, \quad x \in(0, \pi / 2) .
$$

Its hyperbolic version, sometimes called hyperbolic Cusa-Huygens inequality [12], is stated as follows:

$$
\frac{\sinh x}{x}<\frac{2+\cosh x}{3}, \quad x \neq 0 .
$$

Obtaining extended and generalized sharp versions of the above inequalities has been the interest among many researchers in recent years. In $[5,16]$ the following two sided inequality has been obtained:

$$
\left(\frac{2+\cos x}{3}\right)^{a}<\frac{\sin x}{x}<\frac{2+\cos x}{3}, \quad x \in(0, \pi / 2),
$$

with the best positive constants $a \approx 1.11374$ and 1 .

Sándor and Oláh-Gál [18, Theorems 1 and 2] proved the inequalities

$$
\frac{2+\cos x}{\pi}<\frac{\sin x}{x}<\frac{2+(\pi / 2) \cos x}{\pi}, \quad x \in(0, \pi / 2) .
$$

Received January 7, 2019.

2010 Mathematics Subject Classification. 26D05, 26D07, 26D15.

Key words and phrases. Cusa-Huygens inequality, trigonometric-polynomial bounds, hyperbolic version, monotonicity, Yang's inequality.

https://doi.org/10.12697/ACUTM.2020.24.12

Corresponding author: Christophe Chesneau 
For $x \in(-\pi / 2, \pi / 2)$, the double inequality

$$
\frac{p-1+\cos x}{p}<\frac{\sin x}{x}<\frac{2+\cos x}{3},
$$

where $p \approx 2.75194$, is due to Bhayo et al. [3]. Left inequality in (1.4) is sharper than the corresponding inequalities in (1.2) and (1.3). For $x \in$ $(0, \pi / 2)$, the two sided inequality

$$
\frac{p-1+\cos x}{p}<\frac{\sin x}{x}<\frac{2+(4 / \pi) \cos x}{\pi},
$$

where $p \approx 2.75194$, refines (1.3). It gives an optimal answer to the Oppenheim's problem [13]. It is already discussed in [4] and proved by Qi et al. [15] in a more general case.

On the other side, as a natural approach, Mortici [11] established trigonometric-polynomial bounds for $\sin x / x$ :

$$
\frac{2+\cos x}{3}-\frac{x^{4}}{180}<\frac{\sin x}{x}<\frac{2+\cos x}{3}-\frac{x^{4}}{180}+\frac{x^{6}}{3780}, \quad x \in(0, \pi / 2) .
$$

Recently, Malešević et al. [9] extended and generalized (1.6) as follows. If $x \in(0, \pi / 2)$ and $n$ is a natural number, then

$$
\begin{aligned}
& \frac{2+\cos x}{3}+\sum_{k=2}^{2 n}(-1)^{k+1} B(k) x^{2 k}<\frac{\sin x}{x} \\
& <\frac{2+\cos x}{3}+\sum_{k=2}^{2 n+1}(-1)^{k+1} B(k) x^{2 k}
\end{aligned}
$$

where $B(k)=2(k-1) /[3(2 k+1) !]$.

Putting $n=1$ in (1.7), the authors of the paper [9] obtain (1.6). Putting $n=2$, they obtain for $x \in(0, \pi / 2)$ the inequalities

$$
\begin{aligned}
& \frac{2+\cos x}{3}-\frac{x^{4}}{180}+\frac{x^{6}}{3780}-\frac{x^{8}}{181440}<\frac{\sin x}{x} \\
& <\frac{2+\cos x}{3}-\frac{x^{4}}{180}+\frac{x^{6}}{3780}-\frac{x^{8}}{181440}+\frac{x^{10}}{14968800} .
\end{aligned}
$$

In this paper, we give simpler alternative proofs of (1.4) and (1.5) as well as establish new extended refined forms of the inequalities listed above. We also aim to improve Yang's inequality [19] in the last section.

\section{Preliminaries and lemmas}

We start by recalling the power series expansions

$$
\sin x=\sum_{k=0}^{\infty} \frac{(-1)^{k}}{(2 k+1) !} x^{2 k+1}, \quad x \in \mathbb{R},
$$


and

$$
\cos x=\sum_{k=0}^{\infty} \frac{(-1)^{k}}{(2 k) !} x^{2 k}, \quad x \in \mathbb{R} .
$$

The following lemmas will be applied to prove the main results of this paper.

Lemma 1 (The l'Hôspital monotonicity rule [1]). Let $f_{1}, f_{2}:[p, q] \rightarrow \mathbb{R}$ be two continuous functions which are derivable on $(p, q)$ and $f_{2}^{\prime}(x) \neq 0$ for any $x \in(p, q)$. Let

$$
A(x)=\frac{f_{1}(x)-f_{1}(p)}{f_{2}(x)-f_{2}(p)} \text { and } B(x)=\frac{f_{1}(x)-f_{1}(q)}{f_{2}(x)-f_{2}(q)} .
$$

If $f_{1}^{\prime} / f_{2}^{\prime}$ is increasing (or decreasing) on $(p, q)$, then the functions $A(x)$ and $B(x)$ are also increasing (or decreasing) on $(p, q)$. The strictness of the monotonicity in the conclusion depends on the strictness of the monotonicity of $f^{\prime} / g^{\prime}$.

From Lemma 1 we immediately get the following result.

Lemma 2. For $q>0$ and $m \geqslant 1$ let $f_{1}, f_{2}:(0, q) \rightarrow \mathbb{R}$ be continuous and $m$-derivable functions such that $f_{i}^{(k)}(i=1,2 ; k=1, \ldots, m-1)$ are continuous. Suppose that $f_{2}^{(k)}(x) \neq 0$ for $k=1, \ldots, m$ and $x \in(0, q)$. If $f_{i}(0)=f_{i}^{\prime}(0)=\cdots=f_{i}^{(m-1)}(0)=0(i=1,2)$ and $f_{1}^{(m)} / f_{2}^{(m)}$ is increasing (or decreasing) on $(0, q)$, then the function $f_{1}(x) / f_{2}(x)$ is also increasing (or decreasing) on $(0, q)$.

Lemma 3 (see [2], Lemma 2). The function $H(x)=\frac{\sin x-x \cos x}{x^{2} \sin x}$ is strictly positive increasing in $(0, \pi / 2)$.

Lemma 4. The function $\phi(x)=\frac{\sinh x-x \cosh x}{x^{2} \sinh x}$ is strictly negative increasing for $x>0$.

Proof. The function $\phi(x)$ is clearly negative as $\sinh x / x<\cosh x$ for $x>0$ (see [10]). Let us now set

$$
\phi(x)=\frac{\sinh x-x \cosh x}{x^{2} \sinh x}=\frac{\phi_{1}(x)}{\phi_{2}(x)} .
$$

Here the functions $\phi_{1}(x)=\sinh x-x \cosh x$ and $\phi_{2}(x)=x^{2} \sinh x$ satisfy the conditions of Lemma 2 with $m=2$, and

$$
\frac{\phi_{1}^{\prime \prime}(x)}{\phi_{2}^{\prime \prime}(x)}=\frac{-\cosh x}{x \sinh x+3 \cosh x}=\frac{-1}{x \tanh x+3} .
$$

It is well known that $x \tanh x$ is increasing, implying that $x \tanh x+3$ is increasing. By Lemma $2, \phi(x)$ is a strictly increasing function for $x>0$. 


\section{Main results and proofs}

We first give a simpler alternative proof of (1.4).

Theorem 1 (see [3], Theorem 1). If $x \in(-\pi / 2, \pi / 2)$, then

$$
\frac{2+(\pi-2) \cos x}{\pi}<\frac{\sin x}{x}<\frac{2+\cos x}{3} .
$$

Proof. It is enough to prove the result for $x \in(0, \pi / 2)$. Let us set

$$
f(x)=\frac{\frac{\sin x}{x}-\cos x}{\cos x-1}=\frac{f_{1}(x)}{f_{2}(x)}
$$

where $f_{1}(x)=\sin x / x-\cos x$ and $f_{2}(x)=\cos x-1$ satisfy $f_{1}(0+)=0$ and $f_{2}(0)=0$. Since

$$
\frac{f_{1}^{\prime}(x)}{f_{2}^{\prime}(x)}=\frac{\frac{x \cos x-\sin x}{x^{2}}+\sin x}{-\sin x}=\frac{\sin x-x \cos x}{x^{2} \sin x}-1,
$$

by Lemmas 1 and $3, f(x)$ is strictly increasing in $(0, \pi / 2)$. We end the proof with $f(0+)=-2 / 3$ and $f(\pi / 2)=-2 / \pi$.

In the next result, we extend the inequality (1.1).

Theorem 2. If $x \in(0, \lambda)$ with $\lambda>0$, then

$$
-\delta+(1+\delta) \cosh x<\frac{\sinh x}{x}<\frac{2+\cosh x}{3},
$$

where $\delta=(\sinh \lambda / \lambda-\cosh \lambda) /(\cosh \lambda-1)$.

Proof. Let us consider

$$
f(x)=\frac{\frac{\sinh x}{x}-\cosh x}{\cosh x-1}=\frac{f_{1}(x)}{f_{2}(x)}
$$

where $f_{1}(x)=\sinh x / x-\cosh x$ and $f_{2}(x)=\cosh x-1$ with $f_{1}(0+)=0$ and $f_{2}(0)=0$. We have

$$
\frac{f_{1}^{\prime}(x)}{f_{2}^{\prime}(x)}=\frac{x \cosh x-\sinh x}{x^{2} \sinh x}-1 .
$$

By Lemmas 1 and $4, f(x)$ is strictly decreasing in $(0, \lambda)$. The desired result follows from $f(\lambda)=\delta$ and $f(0+)=-2 / 3$.

In the following theorem we present another simple proof of (1.5).

Theorem 3 (se $[4,15])$. If $x \in(0, \pi / 2)$, then

$$
\frac{2+(\pi-2) \cos x}{\pi}<\frac{\sin x}{x}<\frac{2+(4 / \pi) \cos x}{\pi} .
$$


Proof. Let us set

$$
f(x)=\frac{\frac{\sin x}{x}-\frac{2}{\pi}}{\cos x}=\frac{f_{1}(x)}{f_{2}(x)},
$$

where $f_{1}(x)=\sin x / x-2 / \pi$ and $f_{2}(x)=\cos x$ with $f_{1}(\pi / 2)=0$ and $f_{2}(\pi / 2)=0$. The function

$$
\frac{f_{1}^{\prime}(x)}{f_{2}^{\prime}(x)}=\frac{\sin x-x \cos x}{x^{2} \sin x}
$$

is strictly increasing in $(0, \pi / 2)$ by Lemma 3 . So $f(x)$ is also strictly increasing in $(0, \pi / 2)$ by Lemma 1 . Therefore, the proof of Theorem 3 follows with $f(0+)=(\pi-2) / \pi$ and $f(\pi / 2-)=4 / \pi^{2}$ by l'Hôspital's rule.

Motivated by (1.6), we now establish trigonometric-polynomial bounds for $\sin x / x$ with a different approach.

Theorem 4. If $x \in(0, \pi / 2)$, then

$$
\frac{2+\cos x}{3}-\frac{x^{4}}{a}<\frac{\sin x}{x}<\frac{2+\cos x}{3}-\frac{x^{4}}{b}
$$

with the constants $a=180$ and $b \approx 202.618886$.

Proof. Let us set

$$
f(x)=\frac{-3 x^{5}}{3 \sin x-2 x-x \cos x}=\frac{f_{1}(x)}{f_{2}(x)} .
$$

It is not difficult to see that the functions $f_{1}(x)=-3 x^{5}$ and $f_{2}(x)=3 \sin x-$ $2 x-x \cos x$ satisfy the conditions of Lemma 2 with $q=\pi / 2$ and $m=3$. Here we have

$$
\frac{f_{1}^{\prime \prime \prime}(x)}{f_{2}^{\prime \prime \prime}(x)}=180 \frac{x}{\sin x}
$$

which is clearly increasing in $(0, \pi / 2)$. Thus by Lemma 2 , the function $f(x)=$ $f_{1}(x) / f_{2}(x)$ is also increasing in $(0, \pi / 2)$. Since $f(0+)=180$ and $f(\pi / 2) \approx$ 202.618886, we get (3.1).

The hyperbolic version of (3.1) is given in next theorem.

Theorem 5. If $x \in(0, \lambda)$, where $\lambda>0$, then

$$
\frac{2+\cosh x}{3}-\frac{x^{4}}{m}<\frac{\sinh x}{x}<\frac{2+\cosh x}{3}-\frac{x^{4}}{n}
$$

with the constants $m=-3 \lambda^{5} /(3 \sinh \lambda-2 \lambda-\lambda \cosh \lambda)$ and $n=180$.

Proof. The proof is omitted since it is similar to the one of Theorem 4.

A refinement of upper bounds of (1.3) and (1.5) can be seen below. 
Theorem 6. For $x \in(0, \pi / 2)$, one has

$$
\frac{\sin x}{x}<\frac{2+(\pi-2) \cos x}{\pi}+\frac{\pi-3}{3 \pi} x^{2} .
$$

Proof. As in the proofs of previous theorems, let us set

$$
f(x)=\frac{\pi \sin x-2 x-(\pi-2) x \cos x}{\pi x^{3}}=\frac{f_{1}(x)}{f_{2}(x)} .
$$

Here $f_{1}(x)=\pi \sin x-2 x-(\pi-2) x \cos x$ and $f_{2}(x)=\pi x^{3}$ satisfy the conditions of Lemma 2 with $q=\pi / 2$ and $m=3$. We have

$$
\frac{f_{1}^{\prime \prime \prime}(x)}{f_{2}^{\prime \prime \prime}(x)}=\frac{-\pi \cos x+(\pi-2)(\cos x-x \sin x)}{6 \pi}=\frac{f_{3}(x)}{6 \pi}
$$

where

$$
f_{3}(x)=-\pi \cos x+(\pi-2)(3 \cos x-x \sin x) .
$$

The derivative

$$
f_{3}^{\prime}(x)=(-3 \pi+8) \sin x-(\pi-2) x \cos x<0
$$

implies that $f_{3}(x)$ is strictly decreasing in $(0, \pi / 2)$. Therefore, by Lemma 2 $f(x)$ is also decreasing and hence

$$
f(0+)>\frac{\pi \sin x-2 x-(\pi-2) x \cos x}{\pi x^{3}} .
$$

With $f(0+)=(\pi-3) /(3 \pi)$, the result is proved.

Remark 1. There is no strict comparison between the corresponding bounds of (1.5) and (3.2). However when $x$ is close to zero, (3.2) is better since the inequality becomes an equality for $x=0$.

Combining (1.5) and (3.2) we have, for $x \in(0, \pi / 2)$, that

$$
\frac{2+(\pi-2) \cos x}{\pi}<\frac{\sin x}{x}<\frac{2+(\pi-2) \cos x}{\pi}+\frac{\pi-3}{3 \pi} x^{2} .
$$

In the following theorem, we extend and refine the bounds of (3.3).

Theorem 7. If $n \in \mathbb{N}$ (the set of natural numbers) and $x \in(0, \pi)$, then we have

$$
M(x)<\frac{\sin x}{x}<N(x)
$$

where

$$
M(x)=\frac{2+(\pi-2) \cos x}{\pi}+\frac{2}{\pi} \sum_{k=1}^{2 n}(-1)^{k+1} \frac{(k \pi-2 k-1)}{(2 k+1) !} x^{2 k}
$$

and

$$
N(x)=\frac{2+(\pi-2) \cos x}{\pi}+\frac{2}{\pi} \sum_{k=1}^{2 n+1}(-1)^{k+1} \frac{(k \pi-2 k-1)}{(2 k+1) !} x^{2 k} .
$$


Proof. Let us set

$$
f(x)=\frac{\sin x}{x}-M(x) .
$$

Utilizing (2.1) and (2.2), we get

$$
\begin{aligned}
f(x)= & \sum_{k=0}^{\infty}(-1)^{k} \frac{x^{2 k}}{(2 k+1) !}-\frac{2}{\pi}-\frac{\pi-2}{\pi} \sum_{k=0}^{\infty}(-1)^{k} \frac{x^{2 k}}{(2 k) !} \\
& -\frac{2}{\pi} \sum_{k=1}^{2 n}(-1)^{k+1} \frac{k \pi-2 k-1}{(2 k+1) !} x^{2 k} \\
= & \frac{2}{\pi} \sum_{k=0}^{\infty}(-1)^{k+1} \frac{k \pi-2 k-1}{(2 k+1) !} x^{2 k}-\frac{2}{\pi}-\frac{2}{\pi} \sum_{k=1}^{2 n}(-1)^{k+1} \frac{k \pi-2 k-1}{(2 k+1) !} x^{2 k} \\
= & -\frac{2}{\pi} R,
\end{aligned}
$$

where

$$
R=\sum_{k=2 n+1}^{\infty}(-1)^{k} \frac{k \pi-2 k-1}{(2 k+1) !} x^{2 k} .
$$

Hence $R$ can be viewed as a rest of the alternating series $S$ given by

$$
S=\sum_{k=2}^{\infty}(-1)^{k} a_{k},
$$

with $a_{k}=[(k \pi-2 k-1) /(2 k+1) !] x^{2 k}>0$. Now for $k \geqslant 2$ and $x \in(0, \pi)$ we have

$$
(k \pi-2 k-1)\left(4 k^{2}+10 k+6-x^{2}\right) \geqslant(2 \pi-5)\left(42-\pi^{2}\right)>(\pi-2) x^{2} .
$$

This gives

$$
\frac{k \pi-2 k-1}{(2 k+1) !} x^{2 k}>\frac{(k+1) \pi-2(k+1)-1}{(2 k+3) !} x^{2(k+1)} .
$$

Hence $\left|a_{k}\right|>\left|a_{k+1}\right|$. Moreover, for $x \in(0, \pi)$ we have

$$
\lim _{k \rightarrow \infty}\left|a_{k}\right|=\lim _{k \rightarrow \infty} \frac{k \pi-2 k-1}{(2 k+1) !} x^{2 k}=\frac{\pi-2}{2} \lim _{k \rightarrow \infty} \frac{x^{2 k}}{(2 k) !}=0 .
$$

This implies that $S$ is convergent and, by a special result on alternating series, $R$ has the same sign to the first term of its sum, i.e., $(-1)^{2 n+1} a_{2 n+1}<$ 0 . So $R<0$, implying that $f(x)>0$, and

$$
M(x)<\frac{\sin x}{x} .
$$


Similarly, set

$$
\begin{aligned}
g(x) & =N(x)-\frac{\sin x}{x} \\
& =\frac{2+(\pi-2) \cos x}{\pi}+\frac{2}{\pi} \sum_{k=1}^{2 n+1}(-1)^{k+1} \frac{k \pi-2 k-1}{(2 k+1) !} x^{2 k}-\frac{\sin x}{x} .
\end{aligned}
$$

Using again (2.1) and (2.2) and proceeding as in the case of $f(x)$, we obtain

$$
g(x)=\frac{2}{\pi} R_{*},
$$

where

$$
R_{*}=\sum_{k=2 n+2}^{\infty}(-1)^{k} \frac{k \pi-2 k-1}{(2 k+1) !} x^{2 k} .
$$

Let us observe that $R_{*}$ is a rest of the alternating series $S$. By applying similar arguments to $R$, we get $R_{*}>0$, implying that $g(x)>0$ and hence

$$
\frac{\sin x}{x}<N(x) \text {. }
$$

The proof follows from (3.5) and (3.6).

Some particular cases of Theorem 7 are presented below. If $x \in(0, \pi)$, then by putting $n=1$ in (3.4) we get

$$
\begin{aligned}
& \frac{2+(\pi-2) \cos x}{\pi}+\frac{2}{\pi}\left[\frac{\pi-3}{3 !} x^{2}-\frac{2 \pi-5}{5 !} x^{4}\right]<\frac{\sin x}{x} \\
& <\frac{2+(\pi-2) \cos x}{\pi}+\frac{2}{\pi}\left[\frac{\pi-3}{3 !} x^{2}-\frac{2 \pi-5}{5 !} x^{4}+\frac{3 \pi-7}{7 !} x^{6}\right] .
\end{aligned}
$$

By putting $n=2$ in (3.4) we get

$$
\begin{aligned}
& \frac{2+(\pi-2) \cos x}{\pi}+\frac{2}{\pi}\left[\frac{\pi-3}{3 !} x^{2}-\frac{2 \pi-5}{5 !} x^{4}+\frac{3 \pi-7}{7 !} x^{6}-\frac{4 \pi-9}{9 !} x^{8}\right] \\
& <\frac{\sin x}{x}<\frac{2+(\pi-2) \cos x}{\pi} \\
& \quad+\frac{2}{\pi}\left[\frac{(\pi-3)}{3 !} x^{2}-\frac{2 \pi-5}{5 !} x^{4}+\frac{3 \pi-7}{7 !} x^{6}-\frac{4 \pi-9}{9 !} x^{8}+\frac{5 \pi-11}{11 !} x^{10}\right] .
\end{aligned}
$$

Next we claim that, the generalized extended bounds for $\sin x / x$ in the following theorem are sharper than the corresponding bounds in (1.7).

Theorem 8. If $m=2 n-1$, where $n \in \mathbb{N}$ and $x \in(0, \pi)$, then we have

$$
F(x)<\frac{\sin x}{x}<G(x),
$$


where

$$
F(x)=\frac{2 m+\cos x}{2 m+1}+\frac{2}{2 m+1} \sum_{k=1}^{m+1} \frac{k-m}{(2 k+1) !}(-1)^{k+1} x^{2 k}
$$

and

$$
G(x)=\frac{(2 m+2)+\cos x}{2 m+3}+\frac{2}{2 m+3} \sum_{k=1}^{m+2} \frac{k-m-1}{(2 k+1) !}(-1)^{k+1} x^{2 k} .
$$

Proof. Let us set

$$
\begin{aligned}
f(x) & =\frac{\sin x}{x}-F(x) \\
& =\frac{\sin x}{x}-\frac{2 m}{2 m+1}-\frac{1}{2 m+1} \cos x-\frac{2}{2 m+1} \sum_{k=1}^{m+1} \frac{k-m}{(2 k+1) !}(-1)^{k+1} x^{2 k} .
\end{aligned}
$$

Utilizing (2.1) and (2.2), after some calculations, we get

$$
\begin{aligned}
f(x)= & \sum_{k=0}^{\infty} \frac{(-1)^{k}}{(2 k+1) !} x^{2 k}-\frac{2 m}{2 m+1}-\frac{1}{2 m+1} \sum_{k=0}^{\infty} \frac{(-1)^{k}}{(2 k) !} x^{2 k} \\
& -\frac{2}{2 m+1} \sum_{k=1}^{m+1} \frac{k-m}{(2 k+1) !}(-1)^{k+1} x^{2 k} \\
= & \frac{2}{2 m+1} \sum_{k=m+2}^{\infty} \frac{k-m}{(2 k+1) !}(-1)^{k+1} x^{2 k}=\frac{2}{2 m+1} x^{2 m} T,
\end{aligned}
$$

where

$$
T=\sum_{k=2}^{\infty} \frac{k}{(2 k+2 m+1) !}(-1)^{k} x^{2 k}
$$

Then $T$ can be viewed as a rest of the alternating series

$$
U=\sum_{k=1}^{\infty}(-1)^{k} b_{k}
$$

with $b_{k}=(k /(2 k+2 m+1) !) x^{2 k}$. Now, for $k \geqslant 1$ and $x \in(0, \pi)$, we have

$$
k\left[4(k+m)^{2}+10(k+m)+6\right]-k x^{2} \geqslant k\left(42-\pi^{2}\right)>x^{2} .
$$

This gives

$$
\frac{k}{(2 k+2 m+1) !} x^{2 k}>\frac{k+1}{(2 k+2 m+3) !} x^{2 k+2} .
$$

Hence $\left|b_{k}\right|>\left|b_{k+1}\right|$. Moreover, for $x \in(0, \pi)$, we have

$$
\lim _{k \rightarrow \infty}\left|b_{k}\right|=\lim _{k \rightarrow \infty} \frac{k}{(2 k+2 m+1) !} x^{2 k}=\frac{1}{2} \lim _{k \rightarrow \infty} \frac{x^{2 k}}{(2 k+2 m) !}=0 .
$$


This implies that $U$ is convergent and $(-1)^{2} b_{2}>0$, so $T>0$. Thus, $f(x)>0$ and

$$
F(x)<\frac{\sin x}{x} .
$$

Similarly, by setting $g(x)=G(x)-\frac{\sin x}{x}$, proceeding as in the case of $f(x)$ and applying the same arguments, we get

$$
\frac{\sin x}{x}<G(x)
$$

The proof follows from (3.8) and (3.9).

Some particular cases of Theorem 8 are presented below. If $x \in(0, \pi)$, then by putting $n=1$ (so $m=1$ ) in (3.7) we obtain

$$
\frac{2+\cos x}{3}-\frac{x^{4}}{180}<\frac{\sin x}{x}<\frac{4+\cos x}{5}-\frac{x^{2}}{15}+\frac{x^{6}}{12600} .
$$

By putting $n=2$ (so $m=3$ ) in (3.7) we get

$$
\begin{aligned}
& \frac{6+\cos x}{7}-\frac{2 x^{2}}{21}+\frac{x^{4}}{420}-\frac{x^{8}}{1270080}<\frac{\sin x}{x} \\
& <\frac{8+\cos x}{9}-\frac{x^{2}}{9}+\frac{x^{4}}{270}-\frac{x^{6}}{22680}+\frac{x^{10}}{179625600} .
\end{aligned}
$$

For comparison between particular cases, it can be verified by any mathematical software that the bounds of $\sin x / x$ in (3.10) and (3.11) are sharper than the corresponding bounds in (1.6) and (1.8), respectively. Moreover, all the bounds are trigonometric-polynomial. Therefore, considering the number of terms and the degrees of polynomials in respective bounds, we conclude that our bounds are better.

\section{An application}

Well-known Yang's inequality [19] can be stated as follows. If $A_{1}>$ $0, A_{2}>0, A_{1}+A_{2} \leqslant \pi$, and $0 \leqslant \lambda \leqslant 1$, then

$$
\cos ^{2} \lambda A_{1}+\cos ^{2} \lambda A_{2}-2 \cos \lambda A_{1} \cos \lambda A_{2} \cos \lambda \pi \geqslant \sin ^{2} \lambda \pi .
$$

Inequality (4.1) and its generalizations play an important role in the theory of distribution of values. Therefore, many refinements of (4.1) are established so far. For more details we refer the reader to $[6,8,14,20]$ and the references therein.

Recently Chen and Elezović [6] obtained an improved form of Yang's inequality. Let $A_{i}>0$ with $\sum_{i=1}^{n} A_{i} \leqslant \pi, n \in \mathbb{N}, n>1$, and $0 \leqslant \mu \leqslant 1$. Then

$$
N(\mu) \leqslant \sum_{1 \leqslant i<j \leqslant n} H_{i j} \leqslant M(\mu),
$$


where

and

$$
\begin{gathered}
H_{i j}=\cos ^{2} \mu A_{i}+\cos ^{2} \mu A_{j}-2 \cos \mu A_{i} \cos \mu A_{j} \cos \mu \pi, \\
N(\mu)=\frac{n(n-1)}{2} \pi^{2}\left[\frac{1-(\mu / 2)^{2}}{1+(\mu / 2)^{2}}\right]^{2} \mu^{2} \cos ^{2} \frac{\mu \pi}{2}
\end{gathered}
$$

$$
M(\mu)=\frac{n(n-1)}{2} \pi^{2}\left[\frac{1-(\mu / 2)^{3}}{1+2(\mu / 2)^{3}}\right]^{2} \mu^{2} .
$$

Here we refine the Yang's inequality and hence (4.2) by using the tractable bounds of $\sin x / x$.

Theorem 9. Let $A_{i}>0$ with $\sum_{i=1}^{n} A_{i} \leqslant \pi, n \in \mathbb{N}, n>1$, and $0 \leqslant \mu \leqslant 1$. Then

$$
P(\mu) \leqslant \sum_{1 \leqslant i<j \leqslant n} H_{i j} \leqslant Q(\mu)
$$

where

and

$$
P(\mu)=2 n(n-1)\left[1+\frac{(\pi-2)}{2} \cos \frac{\mu \pi}{2}\right]^{2} \mu^{2} \cos ^{2} \frac{\mu \pi}{2}
$$

$$
Q(\mu)=2 n(n-1)\left\{\left[1+\frac{(\pi-2)}{2} \cos \frac{\mu \pi}{2}\right]+\frac{\mu^{2} \pi^{2}(\pi-3)}{24}\right\}^{2} \mu^{2} .
$$

Proof. We substitute $x=\mu \pi / 2$ in (3.3) to get

$$
\mu\left[1+\frac{(\pi-2)}{2} \cos \frac{\mu \pi}{2}\right]<\sin \frac{\mu \pi}{2}
$$

and

$$
\sin \frac{\mu \pi}{2}<\mu\left[1+\frac{(\pi-2)}{2} \cos \frac{\mu \pi}{2}\right]+\frac{\mu^{3} \pi^{2}(\pi-3)}{24} .
$$

Using the inequality (2.13) from [20], for $1 \leqslant i<j \leqslant n$ we have

We deduce that

$$
4 \sin ^{2} \frac{\mu \pi}{2} \cos ^{2} \frac{\mu \pi}{2} \leqslant H_{i j} \leqslant 4 \sin ^{2} \frac{\mu \pi}{2} \text {. }
$$

$$
\begin{aligned}
& 4\left[1+\frac{(\pi-2)}{2} \cos \frac{\mu \pi}{2}\right]^{2} \mu^{2} \cos ^{2} \frac{\mu \pi}{2} \leqslant H_{i j} \\
& \leqslant 4\left\{\left[1+\frac{(\pi-2)}{2} \cos \frac{\mu \pi}{2}\right]+\frac{\mu^{2} \pi^{2}(\pi-3)}{24}\right\}^{2} \mu^{2} .
\end{aligned}
$$

Summing up all the inequalities in (4.4), we get (4.3).

\section{Acknowledgement}

Thanks are due to anonymous referees and editors for their valuable suggestions that improved the paper. 


\section{References}

[1] G. D. Anderson, M. K. Vamanamurthy, and M. Vuorinen, Conformal Invarients, Inequalities and Quasiconformal maps, John Wiley and Sons, New York, 1997.

[2] Y. J. Bagul and C. Chesneau, Some new simple inequalities involving exponential, trigonometric and hyperbolic functions, Cubo 21(1) (2019), 21-35.

[3] B. A. Bhayo, R. Klén, and J. Sándor, New trigonometric and hyperbolic inequalities, Miskolc Math. Notes 18(1) (2017), 125-137.

[4] B. A. Bhayo and J. Sándor, On Carlson's and Shafer's inequalities, Probl. Anal. Issues Anal. 3(21) (2014), 3-15.

[5] C.-P. Chen and W.-S. Cheung, Sharp Cusa and Becker-Stark inequalities, J. Inequal. Appl. 2011 (2011), Article 136, 6 pp.

[6] C.-P. Chen and N. Elezović, Sharp Redheffer-type and Becker-Stark-type inequalities with an application, Math. Inequal. Appl. 21(4) (2018), 1059-1078.

[7] C. Huygens, Oeuvres Completes 1888-1940, Société Hollondaise des Sciences, Haga.

[8] W. D. Jiang and H. Yun, Sharpening of Jordan's inequality and its applications, JIPAM. J. Inequal. Pure Appl. Math. 7(3) (2006), Article 102, 4 pp.

[9] B. Malešević, T. Lutovac, M. Rašajski, and C. Mortici, Extensions of the natural approach to refinements and generalizations of some trigonometric inequalities, Adv. Difference Equ. 2018 (2018), Paper No. 90, 15 pp.

[10] D. S. Mitrinovic, Analytic Inequalities, Springer-Verlag, Berlin, 1970.

[11] C. Mortici, The natural approach of Wilker-Cusa-Huygens inequalities, Math. Inequal. Appl. 14(3) (2011), 535-541.

[12] E. Neuman and J. Sándor, On some inequalities involving trigonometric and hyperbolic functions with emphasis on the Cusa-Huygens, Wilker and Huygens inequalities, Math. Inequal. Appl. 13(4) (2010), 715-723.

[13] A. Oppenheim, E1277, Amer. Math. Monthly, 64(6) (1957), 504.

[14] A. Y. Özban, A new refined form of Jordan's inequality and its applications, Appl. Math. Lett. 19(2) (2006), 155-160.

[15] F. Qi, Q.-M. Luo, and B.-N. Guo, A Simple proof of Oppenheim's double inequality relating to the cosine and sine functions, J. Math. Inequal. 6(4) (2012), 645-654.

[16] J. Sándor, Sharp Cusa-Huygens and related inequalities, Notes Number Theory Discrete Math. 19(1) (2013), 50-54.

[17] J. Sándor and M. Bencze, On Huygen's trigonometric inequality, RGMIA Res. Rep. Coll. 8(3) (2005), Article 14, 4 pp.

[18] J. Sándor and R. Oláh-Gál, On Cusa-Huygens type trigonometric and hyperbolic inequalities, Acta Univ. Sapientiae Math. 4(2) (2012), 145-153.

[19] L. Yang, Distribution of Values and New Research, Science Press, Beijing, 1982. (Chinese)

[20] C.-J. Zhao and L. Debnath, On generalizations of L. Yang's inequality, JIPAM. J. Inequal. Pure Appl. Math. 3(4) (2002), Article 56, 5 pp.

Department of Mathematics, K. K. M. College, Manwath, Dist : ParbHANI(M.S.) - 431505, INDIA

E-mail address: yjbagul@gmail.com

LMNO, University of CAen-Normandie, Caen, France

E-mail address: christophe.chesneau@unicaen.fr 